\title{
10. ON THE APPLICATION OF HANSEN'S METHOD OF

\author{
PARTIAL ANOMALIES TO THE CALCULATION OF \\ PERTURBATIONS IN COMETARY MOTIONS
}

\author{
V. I. SKRIPNICHENKO \\ Institute for Theoretical Astronomy, Leningrad, U.S.S.R.
}

\begin{abstract}
The main points of the method of partial anomalies are described. The method makes it possible to obtain analytical solutions for comets moving in orbits with high eccentricities. The method may be applied, in particular, to systems in which the orbits of the disturbed and disturbing bodies have points equidistant from the Sun. The method is applied to the calculation of the general perturbations by Saturn on P/Tuttle.
\end{abstract}

In order to increase the convergence of the series employed to express the general perturbations of orbits with high eccentricities Hansen (1856) suggested a method that caused the variations of the functions expanded to decrease. This involved division of the orbit of the disturbed body into segments, and in each of the segments the classical variables (the true, eccentric or mean anomalies) were substituted by new ones - partial anomalies - such that as they varied from 0 to $2 \pi$ any coordinate of the disturbed body would vary only within the range corresponding to the boundaries of the segment.

Each segment is thus characterized by its own rapidly convergent series for the coordinates of the disturbed body developed in a single argument - the partial anomaly related to the segment. The coordinates of the disturbing body are expressed in two-argument series; one of the arguments, a partial anomaly, varies from segment to segment; while the other argument $C^{\prime}$, common to all the segments, has the value of the mean anomaly of the disturbing body at the time of passage of the disturbed body through its perihelion. The magnitude of the variation of this argument from revolution to revolution is given by

$$
\Delta C^{\prime}=2 \pi\left(n^{\prime} / n\right),
$$

where $n$ and $n^{\prime}$ are the mean motions of the disturbed and disturbing bodies, respectively.

Two-argument series for the calculation of general perturbations may be obtained on the basis of the above series. If the orbits do not intersect in the segment of the disturbed body orbit, the convergence with respect to the partial anomaly of all the series used for the calculation of perturbations may be arbitrarily accelerated by reducing the segment in size, i.e., by dividing the orbit of the disturbed body into more segments. However, when a segment contains points close to the intersection of the orbits, the convergence of the expansions for the negative powers of the mutual distance with respect to the second argument remains slow even with an arbitrary reduction in the segment size. This requires operating with two-argument series containing 
many terms with respect to one of the variables, and it prevented Hansen from completing his calculations of the perturbations by the Earth on P/Encke.

Those who followed Hansen - Gyldén, Asten, Backlund, and Wittram - tried to by-pass this obstacle and to increase the convergence of the series by using as the second argument, not $C^{\prime}$, but some other variable introduced by means of elliptic functions. However, the increased convergence with respect to the second argument brought about some difficulty in the determination of the constants of integration. Owing to the tremendous amount of computation and great difficulties involved in the use of these complicated series for the calculation of perturbations - should such series have been obtained - the calculations, started by Asten, Backlund, and Wittram, of the general perturbations on P/Encke by Jupiter were never completed. Subsequent attempts at using the method were also abandoned.

The first to make full use of the method was Nacozy (1969), who determined the Earth's perturbations on $\mathrm{P} /$ Encke by means of a computer. At about the same time the method of partial anomalies had been fully mastered by the author, and half of the necessary calculations had been made on the BESM-4 computer (Skripnichenko, 1970). The difference in approach lies in the fact that Nacozy determined the perturbations by means of harmonic analysis, applying it to particular values of the perturbations as soon as they had been calculated, whereas the author carried out all the calculations analytically by manipulation of Fourier series with numerical coefficients. The programme for applying the method of partial anomalies on the BESM-4 was developed, and using the programme the series were calculated for computing the perturbations by Saturn on the motion of $\mathrm{P} /$ Tuttle.

The procedure for performing the calculation was as follows. The initial sets of elements, corresponding to the moment 1885 Oct. 6.5, were as shown in Table I.

TABLE I

Initial elements

\begin{tabular}{ll}
\hline P/Tuttle & Saturn \\
\hline$M=1.73396$ & $M^{\prime}=359.49935$ \\
$\omega=206.77678$ & $\omega^{\prime}=339.89543$ \\
$\Omega=270.54314$ & $\Omega=113.41486$ \\
$i=54.33026$ & $i^{\prime}=2.48610$ \\
$a=5.7422705 \mathrm{AU}$ & $a^{\prime}=9.5692934 \mathrm{AU}$ \\
$e=0.8215463$ & $e^{\prime}=0.0568010$ \\
\hline
\end{tabular}

Saturn's elements for this moment were taken from the collection of major planet elements available in the department of minor planets and comets at the Institute for Theoretical Astronomy. The elements of P/Tuttle for the same moment were obtained by numerical integration, using the computer programme by Belyaev and including the disturbing effects of eight planets: the initial set of the comet's elements, referred to the time of passage through perihelion in September 1885, was taken from Porter (1961).

The comet's orbit was divided into six segments according to the set of elements 
for the moment 1885 Oct. 6.5. Two points of division were selected at the orbit's perihelion and aphelion, respectively. Two other points, corresponding to true anomalies of $160^{\circ}$ and $170^{\circ}$, separate the portion of the ellipse where the distance between the orbits is the shortest. The remaining two points are symmetric to these with respect to the major axis of the ellipse.

On the basis of the same set of elements, the values of the Fourier coefficients for the coordinates of the comet and Saturn, as well as those for the expansion of the square of their mutual distance, were found for each segment, by means of the usual formulae. To save computation time and to simplify application of the method, double harmonic analysis was employed for transition from the latter expansion to series for the negative powers of the mutual distance. This was done to avoid numerical calculations in one argument and analytical calculations in the other (Hansen, 1856), which is a possible alternative. Further, accurate values of negative powers were derived from approximate values by the method described by Brouwer and Clemence (1961). The expansion for the inverse cube of mutual distance in the $160^{\circ}$ $170^{\circ}$ segment contains 10 harmonics in the partial anomaly and 60 harmonics in the second argument, and it includes all the coefficients larger than 0.001 .

These expansions were used to derive the right-hand sides of the differential equations for Hansen's five elements. We have integrated these equations and found integration constants which, when the method of partial anomalies is used, are singleargument series in the second argument $C^{\prime}$. Subsequently, first-order perturbations were obtained in the three Hansen coordinates: $\delta z$ in the mean anomaly, $v$ in the logarithm of the radius vector, and $\delta s$ in the sine of the heliocentric latitude. Particular checks, which are quite easy to find in this case (Asten, 1872), confirm the correctness of the calculations. The series for the perturbations are contained on magnetic tape but are not illustrated here because they are too cumbersome.

The next and final stage will include a thorough comparison of these perturbations with numerical integration. This will enable us to ascertain the time interval over which the series can be used and found reasonably accurate.

\section{References}

Asten, E.: 1872, Mem. Acad. Imp. Sci. St. Petersb. 18, No. 10.

Brouwer, D. and Clemence, G. M.: 1961, Methods of Celestial Mechanics, Academic Press, New York and London.

Hansen, P. A.: 1856, Suppl. Compt. Rend. Acad. Sci. Paris 1, 121.

Nacozy, P. E: 1969, Astron. J. 74, 544.

Porter, J. G.: 1961, Mem. Br. Astron. Assoc. 39, No. 3.

Skripnichenko, V. I.: 1970, Byull. Inst. Teor. Astron. 12, 16. 